\title{
Solitary Plasmacytoma: A Case Report
}

\author{
Neha Choudhary ${ }^{1}$, Jai Chowdhary ${ }^{2}$, Piyush Chandra ${ }^{3}$, Hemant K Mishra ${ }^{4}$
}

\begin{abstract}
Solitary plasmacytoma of bone (SPB), also called osseous plasmacytoma, SPBs have male preponderance and are two times more common in male as compared to females. Approximately 55 years is the median age of patients for SPB.To avoid progression to multiple myeloma, localized radiation therapy is the first-line treatment. A 45-year-old female visited our hospital due to complaint of pain in right hip and right knee. X-ray CT and MRI showed lytic lesions and heterogeneously hypointense on STIR images in the proximal metaphyseal region of the right femur, pathology showed plasmacytoma.

Keywords: Lytic lesion, Magnetic resonance imaging, Multiple myeloma, Plasmacytoma.

Journal of Mahatma Gandhi University of Medical Sciences \& Technology (2021): 10.5005/jp-journals-10057-0189
\end{abstract}

\section{INTRODUCTION}

Plasma cell malignancies are neoplastic proliferation of B-scells. Solitary bone plasmacytoma is a rare immunoproliferative monoclonal disease with localized proliferation of plasma cells. These cancers can be classified as plasmacytoma (localized extramedullary type) and multiple myeloma (MM) (generalized medullary type). Solitary plasmacytoma of bone (SBP) arises from plasma cells of bone marrow (BM), whereas EMP arises from those in the mucosal surfaces. SBP is infrequent, representing $3-7 \%$ of all plasmacytoma. ${ }^{3}$ The median age for SBP is 55 years and involves bones of the axial skeleton (vertebra, skull); involvement of the distal appendicular skeleton below elbow and knee is rare. SBP has a significantly high risk of progression to MM (65-84\% in 10 years). ${ }^{4}$ Solitary plasmacytomas can occur in bone or soft tissue, and it has a high risk of progression to multiple myeloma. Here, we present a patient who had SPB over femur.

\section{Case Description}

A 45-year-old female visited our hospital due to pain in right hip and right leg. Her past medical history included pain in right knee about 2-3 years ago which was not relieved by analgesic. Recently she experienced some pain and noticed some sound coming from her right hip. The pain, which radiated to her lower limbs, was accompanied by numbness. Prolonged standing and sitting were the aggravating factors of the pain. She could not bear ambulation for long periods due to intermittent claudication.

Serum protein electrophoresis shows raised alpha $2(1.03 \mathrm{gm} / \mathrm{dL})$, albumin, globulin, A/G ratio, alpha1 beta, and gamma proteins within normal limit.

On the X-ray pelvis a well-defined lytic lesion is seen in the proximal metaphyseal region of the right femur with involvement of greater trochanter. Thin internal bony septations are seen. There is pathological fracture involving the lesser trochanter. No involvement of head or joint space is seen. The diagnosis made was GCT and advised for CT and biopsy. \begin{tabular}{l}
\hline${ }^{1-4}$ Department of Radio-Diagnosis, Mahatma Gandhi Medical College \\
\& Hospital, Jaipur, Rajasthan, India
\end{tabular}

Corresponding Author: Neha Choudhary, Department of Radio-Diagnosis, Mahatma Gandhi Medical College \& Hospital, Jaipur, Rajasthan, India, Phone: +91 9929546633, e-mail: choudharyneha652@gmail.com

How to cite this article: Choudhary N, Chowdhary J, Chandra P, et al. Solitary Plasmacytoma: A Case Report. J Mahatma Gandhi Univ Med Sci Tech 2021;6(3):97-99.

Source of support: Nil

Conflict of interest: None

On CT scan of pelvis a well-defined lytic lesion is seen in the proximal metaphyseal region of the right femur with involvement of greater trochanter and proximal aspect of neck. Thin internal bony septations are seen. No matrix calcification is seen. The lesion shows a narrow zone of transition. There is pathological fracture involving the lesser trochanter. No involvement of head or joint space is seen. No fluid fluid level is seen within the lesion.

Previous CECT abdomen shows mild hepatomegaly and study reveals there is lytic destructive lesion of size approx. A $38 \times 39 \mathrm{~mm}$ is seen involving the right femur.

On MRI a well-defined lesion appearing heterogeneously hypointense on STIR images is seen in proximal metaphysis of right femur. The lesion shows internal soft tissue components. No chondroid matrix is seen. No matrix calcification is seen. No involvement of head or joint space is seen. No significant extraosseous soft tissue component is seen.

The radiological diagnosis of giant cell tumor or plasmacytoma were made and advised for histopathological correlation (Fig. 1).

Bone marrow examination shows normal cellularity, normoblastic erythropoiesis, normal leucopoiesis, normal megakaryocytic. Approximately $12 \%$ of plasma cells were seen, few mature, mainly immature and plasmablastic type of

(c) The Author(s). 2021 Open Access This article is distributed under the terms of the Creative Commons Attribution 4.0 International License (https://creativecommons. org/licenses/by-nc/4.0/), which permits unrestricted use, distribution, and non-commercial reproduction in any medium, provided you give appropriate credit to the original author(s) and the source, provide a link to the Creative Commons license, and indicate if changes were made. The Creative Commons Public Domain Dedication waiver (http://creativecommons.org/publicdomain/zero/1.0/) applies to the data made available in this article, unless otherwise stated. 


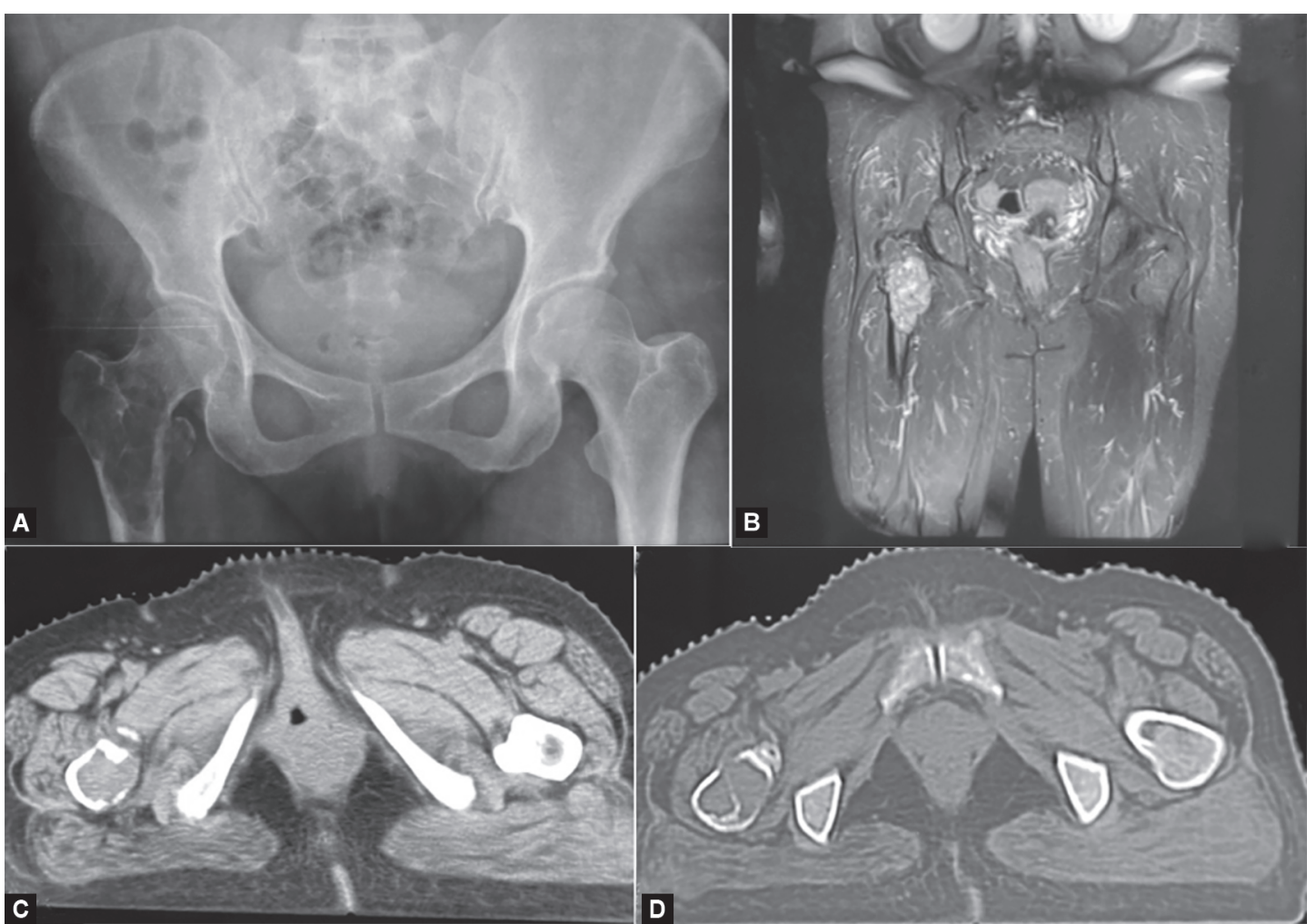

Figs $1 \mathrm{~A}$ and B: X-ray pelvis, axial section of CT and coronal section of MRI shows a well-defined lytic lesion in proximal metaphyseal region of right femur with greater trochanter involvement, thin internal bony septations, and pathological fracture of lesser trochanter, lesion appearing heterogeneously hypointense on STIR

myeloma cells seen. Occasionally flame cells are seen. Few plasma cells show agurophitic granules, binucleate myeloma cells seen.

Serum protein electrophoresis shows raised alpha 2 (1.06 gm/dl), albumin, globulin, low A/G ratio(1.16 gm/dl), alpha1 beta, and gamma proteins within normal limit. Normal protein electrophoresis pattern, paraprotein (monoclonal protein) not detected.

Bence jones protein negative.

Electrophoresis show Alpha peak which is rare in multiple myeloma.

Immunohistochemical study demonstrated cluster of differentiation (CD) $138(+)$, cytokeratins (CK), (-) Kappa light chain (-), Lambda light chain (+), and CD 20 (-) for tumor cells. Urine for myeloma protein was negative. BM aspirate and biopsy were cellular and plasma cells constituted $1-2 \%$ of the marrow nucleated cells.

Based on the above findings, a diagnosis of primary SBP was made.

\section{Discussion}

Solitary bone plasmacytoma or osseous plasmacytoma are the same immunoproliferative monoclonal disease with localized proliferation of plasma cells. It is one type of solitary plasmacytoma. SPB mostly occurs in the flat bones, vertebrae, and the skull; involvement of the extremity bone is rare. ${ }^{1,6,5}$ SPBs have male preponderance and are two times more common in male as compared to females. Approximately 55 years is the median age of patients, which is about 10 years lower than that of patients with multiple myeloma. ${ }^{7,1}$ The disease is rare, approximately 3.5 per 1 million. ${ }^{2}$
The diagnosis requires radiological and histological/ biopsy-proven evidence of the lesion. In radiological diagnosis require plain X-ray $C T$ and $M R I$ of the lesion the plain X-ray shows irregular osteolytic lesion of narrow zone of transition with internal septation. Usually no fracture and soft tissue extension is noted. MRI provides more information of soft tissue extension and its enhancement while CT provides details of the bony part is more advantageous than $\mathrm{CT}$ in terms of enhancement of the mass. The marked homogeneous enhancement on MRI may be helpful for the diagnosis of pelvic SPB. ${ }^{8}$ Radiograph of plasmacytomas shows well-defined lytic lesion is seen in proximal metaphyseal region right femur with involvement of greater trochanter and proximal aspect of neck. Thin internal bony septations are seen. No matrix calcification is seen. The lesion shows a narrow zone of transition. There is pathological fracture involving the lesser trochanter. No involvement of head or joint space is seen.

\section{References}

1. International Myeloma Working Group. Criteria for the classification of monoclonal gammopathies, multiple myeloma and related disorders: a report of the International Myeloma Working Group. Br J Haematol 2003;121(5):749-757. DOI: https://doi.org/10.104 6/j.1365-2141.2003.04355

2. Jawad MU, Scully SP. Skeletal plasmacytoma: progression of disease and impact of local treatment; an analysis of SEER database. J Hematol Oncol 2009;2:41. DOI: 10.1186/1756-8722-2-41

3. Kanthan R, Torkian B. Solitary plasmacytoma of the parotid gland with crystalline inclusions: a case report. World J Surg Oncol 2003;1(1):12. DOI: 10.1186/1477-7819-1-12

4. Kilciksiz S, Karakoyun-Celik O, Agaoglu FY, et al. A review for solitary plasmacytoma of bone and extramedullary plasmacytoma. Scientific World Journal 2012;2012:895765. DOI: 10.1100/2012/895765 
5. Nahi H, Genell A, Walinder G, et al. Incidence, characteristics, and outcome of solitary plasmacytoma and plasma cell leukemia. Population-based data from the Swedish Myeloma Register. Eur J Haematol 2017;99(3):216-222. DOI: 10.1111/ejh.12907

6. Shih LY, Dunn P, Leung WM, et al. Localised plasmacytomas in Taiwan: comparison between extramedullary plasmacytoma and solitary plasmacytoma of bone. Br J Cancer 1995;71(1):128-133. DOI: 10.1038/ bjc.1995.26
7. Soutar R, Lucraft $H$, Jackson $G$, et al. Guidelines on the diagnosis and management of solitary plasmacytoma of bone and solitary extramedullary plasmacytoma. Br J Haematol 2004;124(6):717-726. DOI: 10.1111/j.1365-2141.2004.04834.X

8. Wang Y, Zhu XL, Peeroo MW, et al. Pelvic solitary plasmacytoma: computed tomography and magnetic resonance imaging findings with histopathologic correlation. Korean J Radiol 2015;16(1):146-153. DOI: 10.3348/kjr.2015.16.1.146 